\title{
Computed tomographic correlates of auditory brainstem responses in alcoholics
}

\author{
NAI-SHIN CHU \\ From the Department of Neurology, University of California, Irvine Medical Center, Orange, California, \\ USA
}

SUMMARY A previous study has shown a high incidence of abnormal auditory brainstem responses in alcoholics, particularly when cerebral and/or cerebellar atrophy was present in CT scans. To correlate the electrophysiological findings with definable morphological alterations, quantitative measurements of the brainstem structures in CT scans were made. To determine the relative size of the brainstem, the ratio between the sizes of the brainstem and its cisterns was obtained at the midbrain, upper and lower pons. When compared to the aged-matched control group, the alcoholics as a whole and the alcoholics with abnormal auditory brainstem responses had a decrease in the ratio at all three brainstem levels, but these ratios were not decreased in the alcoholics with the normal auditory brainstem responses. The present data indicate that abnormal brainstem responses in the alcoholics correlated with an increased size of the brainstem cisterns and possible brainstem atrophy.

In our previous study, ${ }^{\prime}$ approximately $40 \%$ of the alcoholics had abnormal auditory brainstem responses. The abnormalities were manifested by a prolongation in the interwave conduction of the auditory brainstem response. One unexpected finding was a high correlation between abnormal auditory brainstem responses and the presence of cerebral cortical and/or cerebellar atrophies in the computed tomographic (CT) scans. There was a 6-fold increase in the incidence of abnormal auditory brainstem responses when the latter conditions were present. This observation raised the possibility that abnormal auditory brainstem response might be related to the atrophic process in the brainstem.

In order to test this hypothesis, CT scans were obtained for quantitative measurements of the brainstem structures and correlated with auditory brainstem response findings. We report that the alcoholics with abnormal auditory brainstem responses had a significant increase in the size of brainstem cisterns indicating possible brainstem atrophy.

Address for reprint requests: Nai-Shin Chu, MD. Department of Neurology, University of California, Irvine Medical Center, 101 City Drive South, Orange, CA 92668, USA.

Received 18 May 1984 and in revised form 6 September 1984. Accepted 18 September 1984

\section{Methods}

Forty-five alcoholics were selected for study. They had history of heavy alcohol drinking from 7 to 50 years with mean of 25 years. The subjects with less than 10 years? history of heavy drinking were those under 35 years of age. Thirteen subjects $(30 \%)$ had been abstinent from alcohol for 2 to 10 years. None of them had neurological disease other than those complicating alcoholism, nor had they had a history of significant head trauma, anoxia-ischaemia, heavy drug abuse, collagen vascular disease, or severe bilateral neurosensory or conductive hearing loss. All had both auditory brainstem responses and CT scans of the head. These tests were not conducted during the acute phases of alcohol-related neurological diseases, that is, acute alcohol intoxication and withdrawal, hepatic encephalopathy, Wernicke's encephalopathy, etc.. In those cases, 6 weeks were allowed for recovery prior to the testing.

The techniques for recording auditory brainstem responses were previously described.' Electrodes were attached at the vertex $(\mathrm{Cz})$ and mastoids, with the ipsilateral mastoid serving as the reference and the contralateral mastoid as the ground. Monaural auditory stimuli consisting of condensation clicks of $0.1 \mathrm{~ms}$ duration and a rate of $11 / \mathrm{Hz}$ were delivered through TDM 39 earphones. The standard intensity was $65 \mathrm{~dB}$ above normal human hearing level. Occasionally, higher intensities of 75 or $85 \mathrm{~dB}$ were used when the components of the auditory brainstem response could not be adequately identified. The far-field electrical activity was amplified by approximately $10^{5}$ with a bandpass of 100 to $3 \mathrm{KH}$ and averaged over 2000 click 

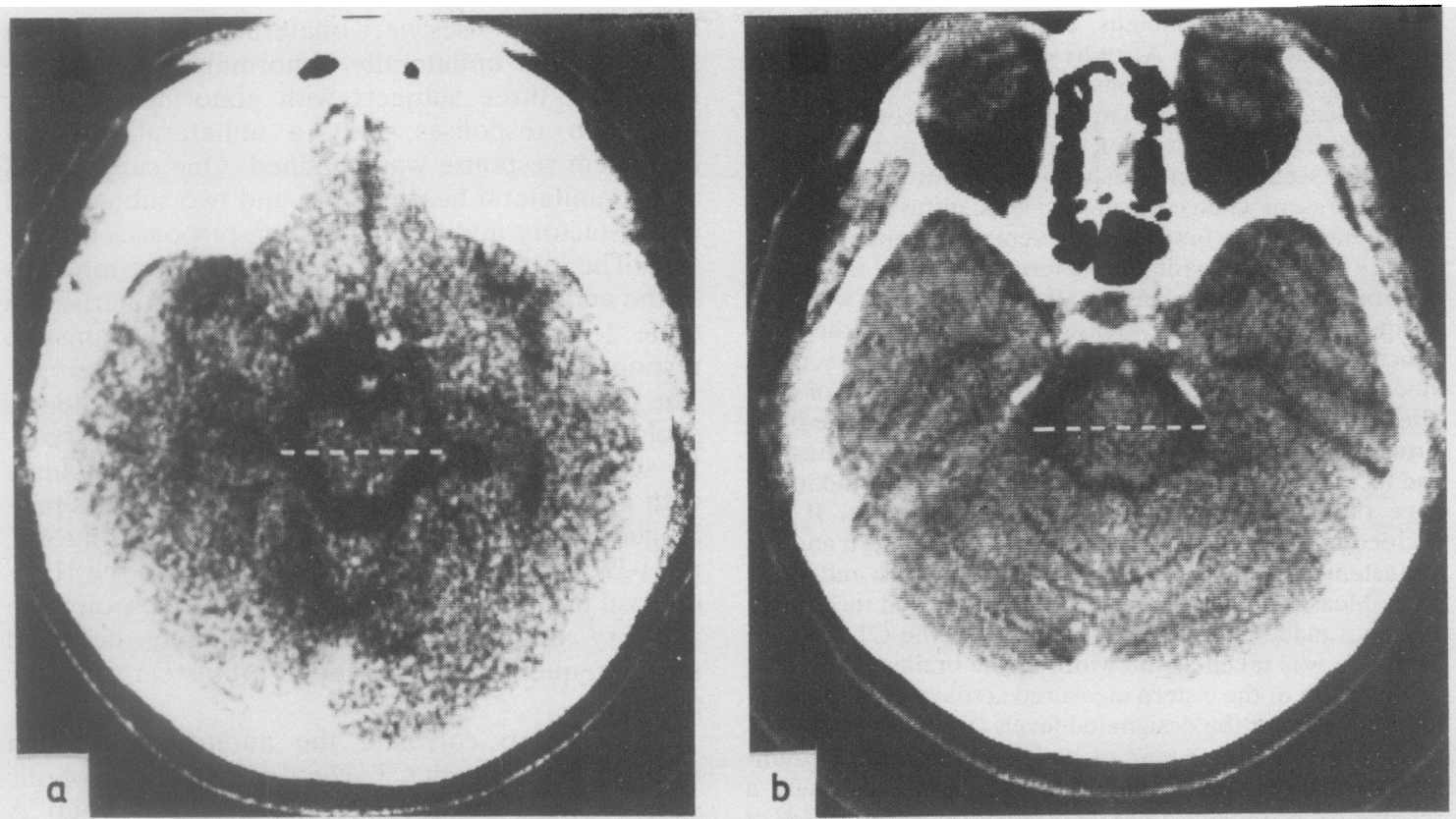

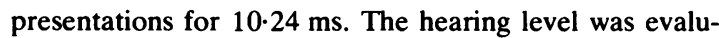
ated by an intensity-latency function of Wave $\mathrm{V}$ at a stimulation rate of $70 / \mathrm{s}$ and click intensities of 35 to $75 \mathrm{~dB}$.

The auditory brainstem response was considered abnormal when the Wave I-V, I-III, and III-V intervals were respectively equal to or greater than $4.5 \mathrm{~ms}, 2.6 \mathrm{~ms}$, and $2.4 \mathrm{~ms}$ (mean $\pm 2.5 \mathrm{SD}$ ) for subjects under 50 years of age, and $4.6 \mathrm{~ms}, 2.7 \mathrm{~ms}$, and $2.3 \mathrm{~ms}$ (mean $\pm 3.0 \mathrm{SD}$ ) for subjects above age of 50 years old. ${ }^{2-7}$ If Wave I could not be identified, the subjects were excluded from the study.

GE Model $8800 \mathrm{CT}$ scanner was used for morphological evaluation of the brainstem. In addition to the routine views of the head, posterior fossa structures were further evaluated by obtaining $5 \mathrm{~mm}$ sections from the foramen magna to the midbrain at the level of superior colliculus. The CT scans were initially evaluated by the neuroradiologist for clinical report. The CT scans were then coded and reviewed by the author without prior knowledge of the subjects' clinical history and the results of auditory brainstem responses. In the majority of cases, there was a general agreement in visual evaluation of CT scans. When disagreement arose, the CT scans were jointly reviewed and the final conclusions were obtained by mutual agreeement.

Visual evaluation of atrophy in cerebral hemispheres, cerebellum, and brainstem was primarily based on the criteria by Koller et al.$^{15}$ Brainstem atrophy was considered present when the cisterns of the brainstem were diffusely prominent. Included in the evaluation were interpeduncu-

Fig 1 CT scan of a 46-year-old alcoholic with abnormal auditory brainstem responses. The subarachnoid space surrounding the midbrain $(A)$, upper pons $(B)$, and lower pons $(C)$ was enlarged. The dashed line indicates the level of ratio measurement and the width of the cistern. 
lar, quadrigeminal, ambiens, prepontine, and cerebellopontine angle cisterns. Atrophy of the cortex of the cerebellar hemispheres was considered present when hemispheric sulci were visible. Atrophy of the cerebellar vermis was considered present when two or more sulci were seen in midline sections. Cerebral hemispheric atrophy was considered present when cortical sulci were diffusely enlarged, which might be accompanied by ventricular enlargement.

Since it was not possible to determine directly the size of the brainstem by CT scans, a ratio measurement was used for quantitative analysis. Measurements by using the bony structures for reference, that is, the distance between the inner tables, were found to be not feasible because of considerable variations in the shape of the head and the bony structures. Furthermore, the small size of the brainstem and its cisterns relative to the bony structures would introduce rather large errors in ratio measurements. It was decided to measure directly the sizes of the cistern and the brainstem at three levels: midbrain, upper pons, and lower pons. Measurements of medulla oblongata and its cisterns were not made due to poor resolution of the CT scanner. The ratio was taken as the width of the brainstem divided by the width of the cistern measured across the midpoint of the brainstem at the designated levels (fig 1). Only the CT scans with adequate views of both cisterns and the brainstem structures were included in the study. To ensure a reliable measurement, ratio determination was repeated at a later date without knowing the results of previous measurements. The means of the two measurements were used for final analysis. The inter-measurement differences were usually less than $5 \%$. Student's $t$ test was used for statistical analysis.

Control data on ratio measurements were obtained from 45 age-matched subjects who had CT scans usually for dizziness, syncopal episodes, headache, minor head trauma, and ruling out seizures. All of them had normal neurological examinations, and none of them had demonstrable focal lesions in the CT scans.

\section{Results}

Both auditory brainstem response and the CT scan of the head were obtained from 45 alcoholics who had fulfiilled the criteria for selection. Their ages ranged from 20 to 75 years with a mean of $48 \cdot 7$. The male to female ratio was 3 to 1 . Eleven subjects $(25 \%)$ had no history of alcohol-related neurological complications other than intoxication.

Auditory brainstem responses were normal in 21 subjects whose mean age was 46 years. The remaining 24 subjects $(53 \%)$ with a mean age of 51 had abnormal auditory brainstem responses, which showed prolonged interwave intervals without alterations in the wave forms (fig 2). Auditory brainstem responses were abnormal in four of 13 subjects who had been abstinent from drinking, and in 20 of 32 subjects who continued to drink. Thus, the incidence of abnormal auditory brainstem responses was twice higher in the latter group ( $31 \%$ vs $63 \%)$. Auditory brainstem responses were bilaterally abnormal in 10 Z subjects, and unilaterally abnormal in 11 . In the remaining three subjects with abnormal auditory brainstem responses, only a unilateral auditory $\frac{\bar{a}}{\sigma}$ brainstem response was obtained. One subject had $T$ severe unilateral hearing loss, and two subjects had unsatisfactory auditory brainstem responses on one side. The incidence of abnormal interwave intervals of the auditory brainstem response is summarised in table 1. Among 34 abnormal auditory brainstem responses, $27(80 \%)$ had prolonged I-V intervals. The majority of abnormal I-V intervals were due to prolongation in the intervals of I-III, III-V, or both. In seven abnormal auditory brainstem responses with normal I-V interval, the I-III interval was prolonged in two, and the III-V interval in five. In total, the I-III interval was abnormal in 12 , and the III-V interval in 21 , indicating that abnormal responses in auditory brainstem response probably occurred more frequently in the upper brainstem auditory pathways.

In order to correlate the auditory brainstem response results with $\mathrm{CT}$ scan findings, the alcoholic subjects were divided into two groups: Group A with normal auditory brainstem responses and Group B with abnormal auditory brainstem responses. The results of their correlations are pre sented in table 2 . In agreement with previous reports, ${ }^{8-13}$ cerebral atrophy was diffuse, but cere

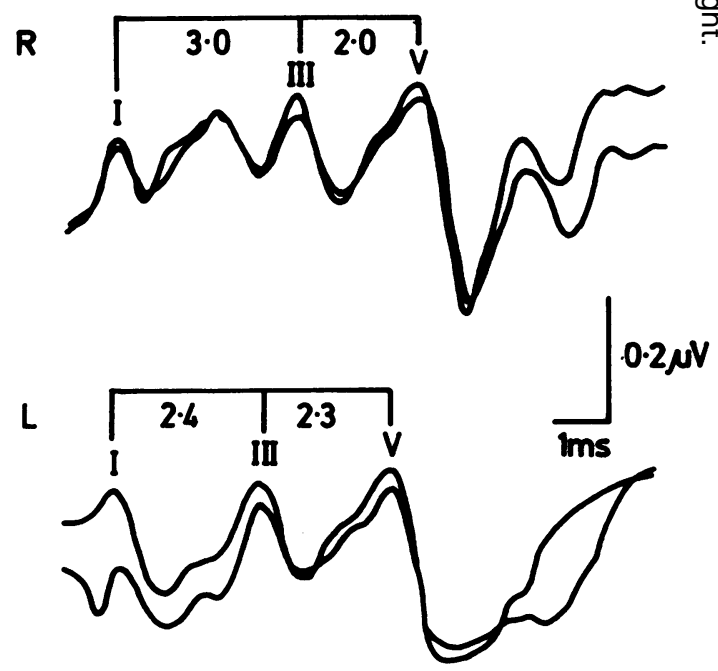

Fig 2 Auditory brainstem evoked responses from the subject whose CT scan was shown in fig 1. The waveform morphology was preserved, but the interwave interval of I-V was bilaterally prolonged. The I-III interval was also prolonged on the right. $R=$ right ear stimulation and $L=$ left ear stimulation. 
Table 1 The incidence of abnormal interwave intervals of the auditory brainstem response in the alcoholics with abnormal responses*

\begin{tabular}{lllll}
\hline & $\begin{array}{l}\text { Normal } \\
\text { I-III and III-V }\end{array}$ & $\begin{array}{l}\text { Abnormal } \\
\text { I-III }\end{array}$ & $\begin{array}{l}\text { Abnormal } \\
\text { III-V }\end{array}$ & $\begin{array}{l}\text { Abnormal } \\
\text { I-III and III-V }\end{array}$ \\
\hline Normal I-V (7) & 0 & 2 & 5 & 0 \\
Abnormal I-V(27) & 5 & 6 & 12 & 4 \\
\hline
\end{tabular}

${ }^{*}$ The numbers are those of the subjects.

Table 2 Brain atrophy in the alcoholics in relation to the age $(A)$ and auditory brainstem evoked responses* $(B)$

\begin{tabular}{|c|c|c|c|c|c|}
\hline $\begin{array}{l}\text { (A) } \\
\text { Age } \\
20-29 \\
30-39 \\
40-49 \\
50-59 \\
60-69 \\
70-79\end{array}$ & $\begin{array}{l}\text { Number of subjects } \\
4 \\
10 \\
12 \\
11 \\
7 \\
1\end{array}$ & $\begin{array}{l}\text { Cereb } \\
1(25 \\
3(30 \\
8(67 \\
7(63 \\
6(86 \\
1(?)\end{array}$ & trophy & $\begin{array}{l}\text { Cerebellar atrophy } \\
1(25 \%) \\
4(40 \%) \\
7(58 \%) \\
8(73 \%) \\
6(86 \%) \\
1(?)\end{array}$ & $\begin{array}{c}\text { Brainstem atrophy } \\
0(0 \%) \\
2(20 \%) \\
6(50 \%) \\
7(63 \%) \\
6(86 \%) \\
1(?)\end{array}$ \\
\hline \multirow{2}{*}{$\begin{array}{l}\text { Total } \\
\text { (B) } \\
\text { Group } \dagger \\
\mathrm{A}=21 \\
\mathrm{~B}=24\end{array}$} & 45 & $26(58$ & & $27(60 \%)$ & $22(49 \%)$ \\
\hline & \multicolumn{2}{|c|}{$\begin{array}{l}\text { Cerebral atrophy } \\
8(38 \%) \\
18(75 \%)\end{array}$} & $\begin{array}{c}\text { Cerebellar atrophy } \\
8(38 \%) \\
19(79 \%)\end{array}$ & $\begin{array}{l}\text { Brainstem atrop } \\
5(24 \%) \\
17(71 \%)\end{array}$ & \\
\hline 45 & \multicolumn{2}{|c|}{$26(58 \%)$} & $27(60 \%)$ & $22(49 \%)$ & \\
\hline
\end{tabular}

*The numbers are those of the subjects.

†Auditory brainstem responses were normal in Group A, but abnormal in Group B.

bellar atrophy was usually restricted to anterior superior vermis and its neighboring areas. In approximately $50 \%$ of the cases, the atrophy was usually mild, but moderate to severe in the remainder. The incidences of cerebral and cerebellar atrophies were twice higher for Group B than for Group A. However, there was an approximately 3 -fold increase in the incidence of brainstem atrophy in the subjects with abnormal auditory brainstem responses, indicating that alcoholics in this group had more selective involvement of the brainstem. It should be noted that when the correlation between auditory brainstem response and CT scan finding was individually evaluated, subjects with brainstem atrophy might have normal auditory brainstem responses, and vice-versa. There was also a progressive increase in the incidence of cerebral, cerebellar, and brainstem atrophies with increasing age (table 2-A). The incidences of atrophy in three selected areas were in general similar for the respective age groups.

Table 3 summarises the results of ratio measurements in 45 alcoholics and 45 age-matched control subjects. The mean age of controls subjects was $47 \cdot 2$ years. When compared with the control group, alcoholics as a whole and the alcoholics with abnormal auditory brainstem responses (Group B) had a highly significant decrease in the ratios $(\mathrm{p}<0.005)$ at all three brainstem levels. However, statistical differences in ratios were not found between control subjects and the alcoholics with normal auditory brainstem responses (Group A) again at all three brainstem levels. Thus, the significant increase in the brainstem cisterns between the control and the alcoholics as a whole was due mainly to the increase from the alcoholics with abnormal auditory brainstem responses.

Table 3 Ratio measurements of the brainstem in the control subjects and the alcoholics*

\begin{tabular}{llll}
\hline & Midbrain & Upper Pons & Lower Pons \\
\hline I. Control (45) $\dagger$ & $0.892 \pm 0.056 \pm$ & $0.876 \pm 0.064$ & $0.832 \pm 0.087$ \\
II. Alcoholic (45) & $0.776 \pm 0.081$ & $0.800 \pm 0.078$ & $0.753 \pm 0.92$ \\
Group A (21) & $0.871 \pm 0.074$ & $0.861 \pm 0.071$ & $0.814 \pm 0.118$ \\
Group B (24) & $0.701 \pm 0.072$ & $0.738 \pm 0.061$ & $0.695 \pm 0.066$ \\
\hline
\end{tabular}

*The ratio was obtained by dividing the width of the brainstem by the width of the cistern at the mid-point level as shown in fig 1 . The numbers are the means and standard deviations.

tThe numbers in the parentheses are those of the subjects. Group A subjects had normal ABRs, whereas Group B had abnormal ABRs. $¥$ The differences in ratios are statistically highly significant $(\mathrm{p}<0.005)$ at all three brainstem levels between the control and the alcoholics as a whole (II). and between Groups $A$ and $B$. The differences between the control and Group A were statistically not significant at all three brainstem levels. 


\section{Discussion}

The auditory brainstem response results in this study are in general agreement with our previous report.' The slightly higher incidence of abnormal auditory brainstem responses in this study as compared to the previous one ( $53 \%$ vs $41 \%$ ) was probably due to the fact that the alcoholics who required CT examination were heavier drinkers who usually had neurological consultation for alcohol-related neurological complications. Thus, the selection of the subjects was biased toward those with neurological complications of alcoholism and the findings may not apply to the alcoholics whose drinking affects only work, family, and social activities.

The incidence of cerebral hemisperhic atrophy in this study is comparable to those reported in most studies. $^{8-12}$ The reported incidences of cerebellar atrophy in alcoholics vary greatly, ranging from $29 \%$ to $92 \% .^{910^{13}}$ In a large series necropsy study, ${ }^{14}$ cerebellar atrophy was found in $\mathbf{2 6 . 8 \%}$ of presumed alcoholics by history. The higher incidence of $49 \%$ in this study might be due partly to biased selection as gait disturbance was the presenting symptom in some of the subjects. It should be emphasised that although brain atrophy was independently assessed, visual evaluation was subjective and the incidences reported here were only approximate.

Although a number of reports have shown high incidence of cerebral and cerebellar atrophies in alcoholics, ${ }^{8-13}$ there was only one report on brainstem atrophy. Koller et al. ${ }^{\text {is }}$ studied 21 chronic alcoholics with CT evidence of cerebellar atrophy. They used the ratio of the width of pre-pontine cistern divided by the distance between the posterior clinoid and the fourth ventricle to indicate the relative size of the brainstem. When compared to the age-matched control, the alcoholics with cerebellar atrophy had a significant increase in the ratio, indicating brainstem atrophy. In our previous report,' and the report by Rosenhammer and Silfveskiold, ${ }^{16}$ there was a high correlation between abnormal auditory brainstem responses and cerebellar atrophy. Thus, those studies seem to indicate that abnormal auditory brainstem responses may be related to brainstem atrophy in alcoholics who have cerebellar atrophy.

The present data demonstrate that alcoholics with abnormal auditory brainstem responses had brainstem atrophy occurring three times higher than those with normal auditory brainstem responses. The quantitative ratio measurements further support subjective visual evaluation of the brainstem by CT scans. There was a highly significant decrease in the brainstem-to-cistern ratios in the alcoholics with abnormal auditory brainstem responses when com- pared to the alcoholics with normal auditory brainstem responses. The significant decrease in the ratios which indicated possible brainstem atrophy was observed at all three brainstem levels, suggesting that brainstem atrophy was generalised.

Caution is needed, however, in interpretation of the results on ratio measurements of the brainstem. Although the decrease in ratio seems to indicate relative decrease in the size of the brainstem, there are inherited uncertainties in the measurements. There are normal variations in the size of the cisterns and the brainstem. The levels and the angles of the head in CT scanning were presumably not exactly the same for every subject. To infer the size of the brainstem by measuring only at one point in one plane imposes difficulty to indicate threedimensional changes.

There are large individual variations in brain sulci and size. ${ }^{14}$ More accurate determination was carried out by Torvik et al, ${ }^{14}$ and Harper and Blumbergs ${ }^{17}$ who had determined the brain weight in alcoholics. Both studies showed a significant decrease in the mean brain weight in alcoholics irrespective whether the brains had the stigma of alcoholic nutritional disease or not. It was concluded that alcoholics had generalised brain atrophy. Unfortunately, the weights of cerebral hemispheres, cerebellum, and the brainstem were not separately measured to sup port such conclusion. In this study, all these struco tures were evaluated by CT scans. Although visuax evaluation of brain atrophy by CT scan needs to be cautious, the findings, nonetheless, indicated that in alcoholics brain atrophy was generalised, and brainstem atrophy is probably as common as cerebral and cerebellar atrophies.

It appears that the increased size of brainstem cisterns or possible brainstem atrophy is one important morpholgical correlate of abnormal auditory brainstem responses in alcoholics. However, brainstem atrophy in CT scan alone is not necessarily associated with abnormal auditory brainstem responses. As mentioned earlier, alcoholics with prominent brainstem cisterns might have normal auditory brainstem responses and vice-viersa. The majority of patients with presumed olivo-pontocerebellar atrophy or spinocerebellar degeneration also have normal auditory brainstem responses. ${ }^{18-20}$ It must be assumed that other pathological processes are also responsible for the high incidence of abnormal auditory brainstem responses in alcohol- $\frac{D}{2}$ ics. Although brainstem lesions in WernickeKorsakoff syndrome, central pontine myelinolysis, $\sigma$ and alcoholic cerebellar degeneration are well $N$ described, ${ }^{21-23}$ systematic or quantitative studies on unselected alcoholics are not available, Pathological study by Lynch $^{24}$ on 11 alcoholics has shown that 
scattered demyelination, gliosis, and neuronal loss in the brainstem were not uncommon. It is possible that brain atrophy including the brainstem results from repeated insults of alcohol intoxication and withdrawal, head trauma, malnutrition, anoxiaischaemia, liver disease, and electrolyte disturbance which are often associated with alcoholism..$^{25}$ Recent clinical and experimental studies ${ }^{26-29}$ have indicated the important role of electrolyte disturbance in widespread demyelination in the brain including the brainstem and the cerebellum which, if not completely recovered, may be partly responsible for abnormal auditory brainstem responses in alcoholics.

In summary, the present quantitative measurements have indicated a high correlation between abnormal auditory brainstem responses and enlarged brainstem cisterns or possible brainstem atrophy. This study also indicates that, in alocholics, brainstem atrophy is probably as common as cerebral and cerebellar atrophies, suggesting generalised atrophy of the brain. This quantitative electrophysiological and morphological study provides evidence that brainstem abnormalities in alcoholics are not as infrequent as previously thiought.

The author is most grateful to Dr Fong Y Tsai, Division of Neuroradiology for his interest in the study and participation in review of the CT scans. This study was supported by a grant from NIAAA (1K02-AA-0049).

\section{References}

' Chu N-S, Squires KC, Starr A. Auditory brainstem responses in chronic alcoholic patients. Electroencephalogr Clin Neurophysiol 1982;54:418-25.

${ }^{2}$ Starr A, Achor J. Auditory brainstem responses in neurological diseases. Arch Neurol 1975;32:761-8.

${ }^{3}$ Beagley HA, Sheldrake JB. Differences in brainstem response latency with age and sex. Br J Audiol 1978; 12:69-77.

${ }^{4}$ Rowe MJ. Normal variability of the brainstem auditory evoked response in young and adult subjects. Electroencephalogr Clin Neurophysiol 1978;44:459-70.

${ }^{5}$ Stockard JE, Stockard JJ, Westmoreland BF, Corfits JL. Brainstem auditory responses. Normal variation as a function of stimulus and subject characteristics. Arch Neurol 1979;36:823-31.

- Chiappa KH, Harrison JL, Brooks EB, Young RR. Brainstem auditory evoked responses in 200 patients with multiple sclerosis. Ann Neurol 1980; 7:135-43.

${ }^{7}$ Rosenhamer HJ, Lindstrom B, Lundborg T. On the use of click-evoked electric brainstem responses in audiological diagnosis. II. The influence of sex and age upon the normal response. Scand Audio 1980;9:93100.

"Epstein PE, Pisani VD, Fawcett JA. Alcoholism and cerebral atrophy. Alcoholism 1977;1:61-65.

${ }^{9}$ Cala LA, Jones B, Mastaglia FL, Wiley B. Brain atrophy and intellectual impairment in heavy drinkers: A clinical, psychometric and computed tomographic study. Aust NZ J Med 1979;8: 147-53.

${ }^{10}$ Bergman H, Borg S, Hindmarch T, Idestrom C-M, Mutzell S. Computed tomography of the brain and neuropsychological assessment of male alcoholic patients and a random sample from the general male population. Acta Psychiat. Scand 1980; Suppl 286: 77-88.

"Lusins J, Zimberg S, Smoker H, Gurley K. Alcoholism and cerebral atrophy: A study of 50 patients with CT scan and psychologic testing. Alcoholism 1980;4:406-11.

12 Ron MA, Acker W, Lishman WA. Computerised tomography of the brain in chronic alcoholism. Brain 1982;105:497-514.

${ }^{13}$ Haubeck A, Lee K. Computed tomography in alcoholic cerebellar atrophy. Neuroradiology 1979;18:77-79.

${ }^{14}$ Torvik A, Lindboe CF, Rogde S. Brain lesions in alcoholics. J Neurol Sci 1982;56:233-48.

is Koller WC, Glatt SL, Perlik S, Huckman MS, Fox JH. Cerebellar atrophy demonstrated by computed tomography. Neurology (NY) 1981;31:405-12.

${ }^{16}$ Rosenhamer HJ, Silfveskiold BP. Slow tremor and delayed brainstem auditory evoked responses in alcoholics. Arch Neurol 1980;37:293-6.

${ }^{17}$ Harper CG, Blumbergs PC. Brain weights in alcoholics. $J$ Neurol Neurosurg Psychiatry 1982;45:838-40.

${ }^{18}$ Fujita M, Hosoki M, Miyazaki M. Brainstem auditory evoked responses in spinocerebellar degeneration and Wilson's disease. Ann Neurol 1981;9:42-47.

${ }^{14}$ Hammond EJ, Wilder BJ. Evoked potentials in olivopontocerebellar atrophy. Arch Neurol 1983;40:366-69.

${ }^{20}$ Gilroy J, Lynn GE. Computerized tomography and auditory-evoked potentials. Arch Neurol 1978;35: 143-7.

21 Victor M, Adams RD, Mancall EL. A restricted form of cerebellar cortical degeneration occurring in alcoholic patients. Arch Neurol 1959; 1:579-688.

${ }^{22}$ Adams RD, Victor M, Mancall EL. Central pontine myelinolysis. Arch Neurol 1959;81:154-72.

${ }^{23}$ Victor M, Adams RD, Collins GH. The WernickeKorsakoff Syndrome. Philadelphia, Davis, 1971.

${ }^{24}$ Lynch MJG. Brain lesions in chronic alcoholism. Arch Pathol 1960;69:342-53.

${ }^{25}$ Victor M. Alcoholism. In: Baker AB and Baker LH eds., Clinical Neurology. Hagerstown: Harper and Row, 1972; Vol. 2, 1-43.

${ }^{26}$ Kleinschmidt-DeMasters BK, Norenberg MD. Rapid correction of hyponatremia causes demyelination: Relation to central pontine myelinolysis. Science 1981;21 1: 1068-70.

${ }^{27}$ Kleinschmidt-DeMasters BK, Norenberg MD. Cerebellar degeneration in the rat following rapid correction of hyponatremia. Ann Neurol 1981; 10:561-5.

${ }^{28}$ Kleinschmidt-DeMasters BK, Norenberg MD. Neuropathologic observations in electrolyte-induced myelinolysis in the rat. $J$ Neuropathol Exp Neurol 1982;41:67-80.

${ }^{29}$ Norenberg MD, Leslie KO, Robertson AS. Association between rise in serum sodium and central pontine myelinolysis. Ann Neurol 1982;11:128-35. 